\title{
Free convection in a triangular enclosure with fluid-saturated porous medium and internal heat generation
}

\author{
Suvash C. Saha ${ }^{1} \quad$ Y. T. Gu ${ }^{2}$ \\ (Received 8 January 2012; revised 10 May 2012)
}

\begin{abstract}
Unsteady natural convection inside a triangular cavity is studied. The cavity is filled with a saturated porous medium has a nonisothermal left inclined wall while the bottom surface is isothermally heated and the right inclined surface is isothermally cooled. Internal heat generation is also considered, which is dependent on the fluid temperature. The governing equations are solved numerically by the finite volume method. The Prandtl number of the fluid is chosen to be 0.7 (air) whereas the aspect ratio and the Rayleigh number are 0.5 and $10^{5}$, respectively. The effect of heat generation on the fluid flow and heat transfer is presented in terms of streamlines and isotherms. The rate of heat transfer through the three surfaces of the enclosure is also presented.
\end{abstract}

http://journal . austms.org.au/ojs/index.php/ANZIAMJ/article/view/5087 gives this article, (c) Austral. Mathematical Soc. 2012. Published May 20, 2012. ISSN 1446-8735. (Print two pages per sheet of paper.) Copies of this article must not be made otherwise available on the internet; instead link directly to this URL for this article. 


\section{Contents}

1 Introduction

C128

2 Mathematical formulation

C129

3 Numerical procedure

C134

4 Results and discussion

C134

5 Conclusion

C137

References

C139

\section{Introduction}

The characteristics of heat and fluid flow for a configuration of isothermal vertical walls, maintained at different temperatures and with adiabatic horizontal walls, are well understood [1,2]. Less work has been carried out for more complex thermal boundary conditions, such as an imposed thermal gradient that is neither purely horizontal nor purely vertical. Shiralkar and Tien [3] numerically investigated the natural convection in an enclosure with temperature gradients imposed in both the horizontal and vertical directions simultaneously. A number of studies related to simultaneously heating and cooling adjacent walls of a square/rectangular enclosure are available $[4,5,6]$. Velusamy et al. [7] investigated the steady two dimensional natural convection flow in a rectangular enclosure with a linearly varying surface temperature on the left vertical wall, cooled right vertical and top walls and a uniformly heated bottom wall. Hossain and Wilson [8] conducted similar work.

In recent decades, flow in a confined porous medium has received considerable attention from researchers working with various experimental, theoretical as well as numerical methodologies. The main application of study in this 
area is developing technology and industry, such as the prevention of sub-oil water pollution, storage of nuclear waste and geothermal energy systems (extensively reviewed by Cheng [10]).

The fluid flow and heat transfer inside a triangular enclosure has been recently considered by many researchers because of its engineering applications in attic shaped buildings, electronic devices, solar collectors and so on. Saha and Khan [11] extensively reviewed natural convection in triangular enclosures. Basak et al. [9] investigated natural convection inside a triangular enclosure with linear heating/cooling on the inclined surfaces. Natural convection in an attic space filled with porous media has also been conducted by a number of researchers $[12,13,14,15]$. In this study, we consider a triangular enclosure filled with porous media and heat generating fluid with non-uniform temperature conditions on one of the sloping walls. A detailed development of the present investigation is given in the subsequent sections.

\section{Mathematical formulation}

Under consideration is a triangular cavity of height $\mathrm{H}$, half length of the base $l$, in a saturated porous medium containing a Newtonian fluid with Prandtl number $\operatorname{Pr}=0.7$ which is initially at rest with a temperature $T_{c}$ (see Figure 1). At time $t=0$, a non-isothermal temperature condition is applied on the left inclined wall where the temperature reduces linearly from bottom to top. The bottom surface is uniformly heated at $T_{h}$ and the top major portion of the right inclined wall is cold at $T_{c}$. The boundary condition at the lower portion of the right inclined wall is explained by the base of the cavity being on a reactor and a small gap at the bottom of the right inclined wall of length $s_{1}=s / 10$ ( $s$ is the total arc length of the right inclined surface) filled with a sodium deposit. The temperature within the gap is expected to vary linearly from $T_{h}$ to $T_{c}$. The effect of temperature dependent heat generation in the flow region is also taken into consideration. The volumetric rate of 


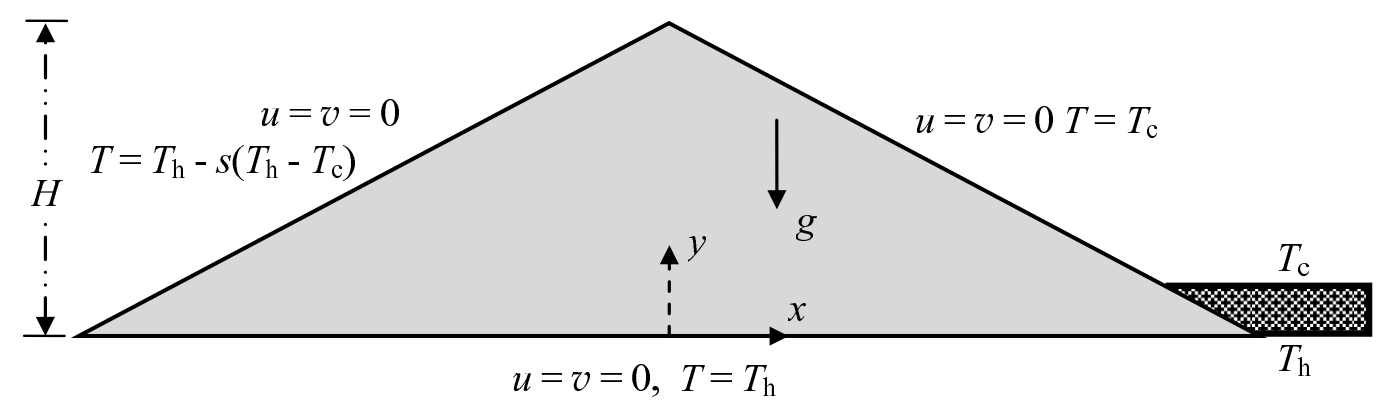

Figure 1: Schematic of the geometry and the coordinate system.

heat generation, $\mathrm{Q}\left(\mathrm{W} / \mathrm{m}^{3}\right)$, is assumed to be

$$
Q= \begin{cases}Q_{0}\left(T-T_{c}\right), & T \geqslant T_{c}, \\ 0, & T \leqslant T_{c},\end{cases}
$$

where $Q_{0}$ is the heat generation constant. The above relation, as explained by Saha et al. [16], is valid as an approximation of the state of some exothermic process, which means that heat flows from the surface to the enclosure. We further assume unsteady laminar flow of a viscous incompressible fluid having constant properties. The effect of buoyancy is included through the well-known Boussinesq approximation. Under the above assumptions, the conservation equations for mass, momentum and energy in a two dimensional Cartesian coordinate system are

$$
\begin{aligned}
& \frac{\partial u}{\partial x}+\frac{\partial v}{\partial y}=0 \\
& \frac{\partial u}{\partial t}+u \frac{\partial u}{\partial x}+v \frac{\partial u}{\partial y}=-\frac{1}{\rho} \frac{\partial p}{\partial x}+v\left(\frac{\partial^{2} u}{\partial x^{2}}+\frac{\partial^{2} u}{\partial y^{2}}\right)-\frac{v u}{K} \\
& \frac{\partial v}{\partial t}+u \frac{\partial v}{\partial x}+v \frac{\partial v}{\partial y}=-\frac{1}{\rho} \frac{\partial p}{\partial y}+v\left(\frac{\partial^{2} v}{\partial x^{2}}+\frac{\partial^{2} v}{\partial y^{2}}\right)-\frac{v v}{K}+g \beta\left(T-T_{c}\right), \\
& \frac{\partial T}{\partial t}+u \frac{\partial T}{\partial x}+v \frac{\partial T}{\partial y}=\kappa\left(\frac{\partial^{2} T}{\partial x^{2}}+\frac{\partial^{2} T}{\partial y^{2}}\right)+\frac{Q_{0}}{\rho C_{p}}\left(T-T_{c}\right)
\end{aligned}
$$


where $u$ and $v$ are the fluid velocity components in the $x$ and $y$-direction, respectively, $t$ is the time, $T$ is the fluid temperature, $p$ is the fluid pressure, $\beta$ is the volumetric thermal expansion coefficient, $K$ is the permeability of the porous medium, and $\rho, \alpha$ and $C_{p}$ are, respectively the density of the fluid, the thermal diffusivity and the specific heat at constant pressure. In the present investigation, porous medium inertia effects are neglected in the momentum equations, and the effects of viscous dissipation are neglected from the energy equation. In Equations (3) and (4), $\mathrm{K}$ is the measure of the permeability of the porous medium (a packed bed of spheres) [8], defined by

$$
\frac{\epsilon^{+3} d^{2}}{180\left(1-\epsilon^{+}\right)^{2}},
$$

where $\mathrm{d}$ is the diameter of the solid sphere, and $\epsilon^{+}$is known as the porosity of the media, defined by

$$
\epsilon^{+}=\frac{V_{f}}{V_{c}} .
$$

Here $V_{f}$ is the volume of the fluid and $V_{c}$ is the control volume. The following dimensionless variables are constructed:

$$
\begin{array}{r}
X=\frac{x}{H}, \quad y=\frac{y}{H}, \quad \tau=\frac{t}{H / U_{0}}, \quad P=\frac{p}{\rho U_{0}}, \\
U=\frac{u}{U_{0}}, \quad V=\frac{v}{U_{0}}, \quad \theta=\frac{T-T_{c}}{T_{h}-T_{c}},
\end{array}
$$

where $\mathrm{U}_{0}=v / \mathrm{H}$ is the reference velocity. Introducing the above dimensionless dependent and independent variables in the governing Equations (3) and (4), 
the following equations are obtained

$$
\begin{aligned}
& \frac{\partial U}{\partial X}+\frac{\partial V}{\partial Y}=0 \\
& \frac{\partial U}{\partial t}+U \frac{\partial U}{\partial X}+V \frac{\partial U}{\partial Y}=-\frac{\partial P}{\partial X}+\operatorname{Pr}\left(\frac{\partial^{2} U}{\partial X^{2}}+\frac{\partial^{2} U}{\partial Y^{2}}-\gamma U\right) \\
& \frac{\partial V}{\partial t}+U \frac{\partial V}{\partial X}+V \frac{\partial V}{\partial Y}=-\frac{\partial P}{\partial Y}+\operatorname{Pr}\left(\frac{\partial^{2} V}{\partial X^{2}}+\frac{\partial^{2} V}{\partial Y^{2}}-\gamma V\right)+\operatorname{Ra} \operatorname{Pr} \theta \\
& \frac{\partial T}{\partial t}+U \frac{\partial \theta}{\partial X}+V \frac{\partial \theta}{\partial Y}=\frac{\partial^{2} \theta}{\partial X^{2}}+\frac{\partial^{2} \theta}{\partial Y^{2}}+\lambda \theta
\end{aligned}
$$

where Rayleigh number

$$
\mathrm{Ra}=\frac{\mathrm{g} \beta\left(\mathrm{T}_{\mathrm{H}}-\mathrm{T}_{\mathrm{C}}\right) \mathrm{H}^{3}}{\mathrm{\kappa} v}, \quad \operatorname{Pr}=\frac{\nu}{\kappa}, \quad \gamma=\frac{\mathrm{H}^{2}}{\mathrm{~K}} \quad \text { and } \quad \lambda=\frac{\mathrm{Q}_{0} \mathrm{H}^{2}}{\rho \nu \mathrm{C}_{\mathrm{p}}} .
$$

The dimensionless initial and boundary conditions are:

- all boundaries are rigid and non-slip;

- at $\tau \leqslant 0, U=V=\theta=0$;

- on the bottom surface, $\theta=1$;

- on the left inclined surface, $\theta=1-s$;

- upper portion of the right inclined surface, $\theta=0$;

- lower portion of the right inclined wall, $\theta=1-s / s_{1}$.

Using the numerically obtained values of the temperature function we obtain the rate of heat flux from each of the walls. The non-dimensional heat flux from any surface is $-\partial T / \partial \mathbf{n}$, where $\mathbf{n}$ is the direction normal to the wall. For example, the non-dimensional heat transfer rate, $\mathrm{Nu}$, per unit length in the depth-wise direction for the left vertical surface is

$$
\mathrm{Nu}=-\int_{0}^{1}\left(\frac{\partial T}{\partial X}\right)_{X=0} d Y .
$$



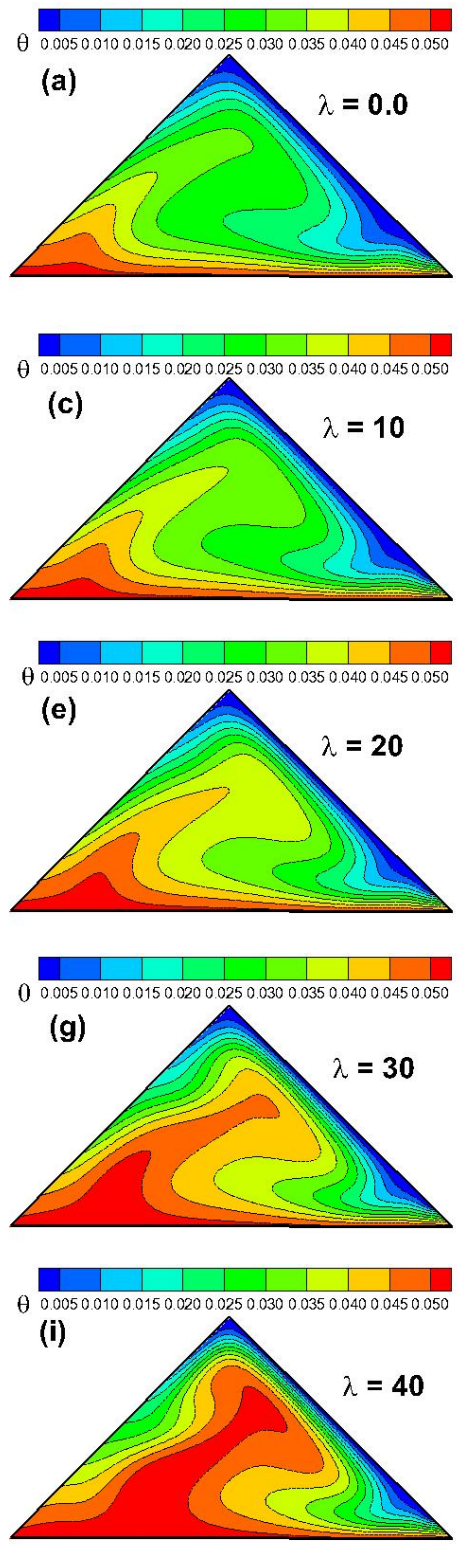
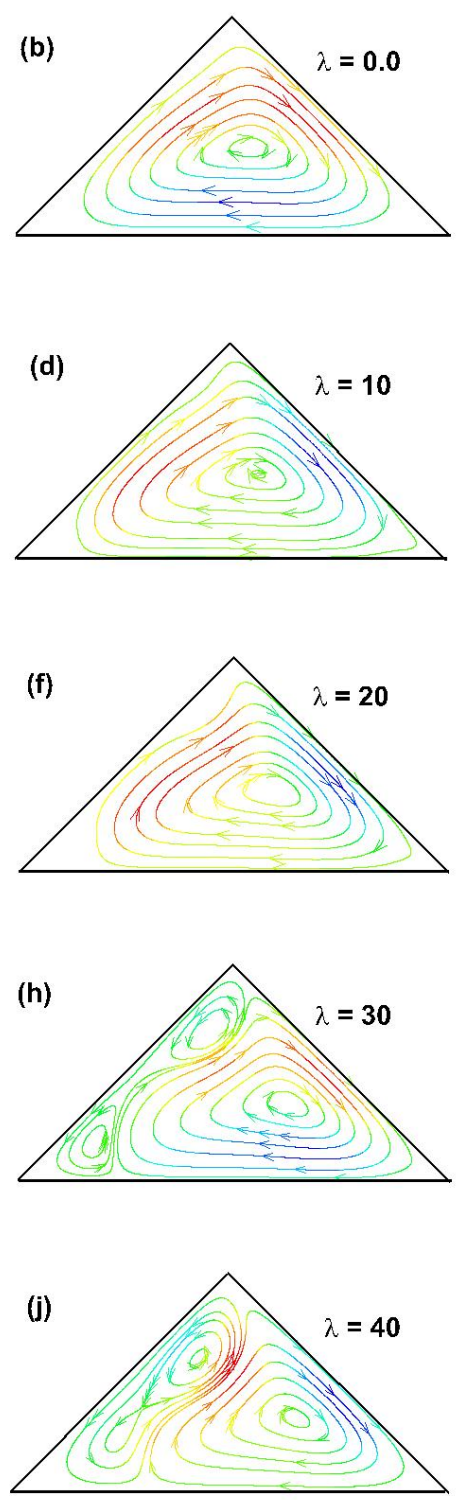

Figure 2: Steady state values of isotherms (left) and velocity field with vector arrow (right): (a-b) $\lambda=0,(\mathrm{c}-\mathrm{d}) \lambda=10,(\mathrm{e}-\mathrm{f}) \lambda=20,(\mathrm{~g}-\mathrm{h}) \lambda=30$, $(\mathrm{i}-\mathrm{j}) \lambda=40$ with parameters $\mathrm{Ra}=10^{5}, \operatorname{Pr}=0.7, \gamma=10.0$. 


\section{Numerical procedure}

Two dimensional numerical simulations were carried out to solve laminar natural convection in a triangular enclosure. For this purpose, an isosceles triangular domain is considered, and a Cartesian coordinate system is adopted with the origin located at the centre of the base, the $x$-axis pointing horizontally, and the $y$-axis pointing vertically. The initial and boundary conditions for the numerical simulations are also specified. That is, the air in the enclosure is initially quiescent and isothermal with a uniform temperature of $\theta=0$. At the time $\tau=0$, the left inclined surface is linearly cooled. On the right inclined wall, the bottom gap is linearly cooled and the top portion is uniformly cooled, and the base of the enclosure is uniformly heated. All three surfaces of the enclosure are assumed to be rigid and non-slip.

The governing equations (9)-(12), along with the specified initial and boundary conditions are solved numerically. The finite volume scheme was chosen to discretize the governing equations, with the QUICK scheme approximating the advection term. The diffusion terms are discretized using central differencing with second order accuracy. A second order implicit time marching scheme was used for the unsteady term. A triangular grid with 8143 nodes was constructed over the domain. By halving the grid size, a detailed grid sensitivity test was carried out to ensure the accuracy of the numerical results. The maximum variation of temperature at a given point inside the enclosure for two grid sizes was observed to be less than $2 \%$. For brevity, the test results of grid dependency are not presented here.

\section{Results and discussion}

Numerical results for natural convection heat transfer for a fluid with internal heat generation in a triangular enclosure, filled with a saturated porous medium with uniform porosity, are described. The left inclined wall is non- 
uniformly cooled and the right inclined and the bottom base are uniformly cooled and heated respectively.

We plot the isotherms and contours of the stream function in Figure 2 for different values of the heat generation parameter $\lambda$, with $\operatorname{Ra}=10^{5}, \operatorname{Pr}=0.7$ and $\gamma=10.0$. Figure 2 represents the isotherms and streamlines for pure fluid in the absence of internal heat generation $(\lambda=0)$. Since the bottom surface is uniformly heated, a heated boundary layer develops adjacent to the bottom surface. Due to the buoyancy effect, the hot fluid inside the boundary layer moves upward from the bottom left tip. The non-uniform heating effect of the left inclined surface causes the hot fluid to move upward and to disperse into the core of the enclosure. On the other hand, the cold fluid near the right slopping wall moves downwards through the cold inclined boundary layer. In the corresponding streamlines, a large convecting cell occupies the whole enclosure with clockwise circulation. For increasing heat generation parameter, $\lambda$, (see Figure 2) the isotherms cluster closer to the right inclined cold surface of the enclosure. The gradient of temperature also appears to increase in the core section of the enclosure. As $\lambda$ increases, the flow becomes stronger and a secondary vortex appears near the left inclined wall. This secondary vortex becomes larger as the heat generation parameter increases. It is expected that the secondary vortex will dominant the flow if the heat generation parameter increases further.

Figures $3(\mathrm{a})-(\mathrm{c})$ plot the total heat transfer along three surfaces of the enclosure for values of the permeability, $0.1 \leqslant \gamma \leqslant 100$, for $\operatorname{Pr}=0.7$ and $\mathrm{Ra}=10^{5}$. These figures show that, as the value of the permeability increases, the heat transfer from the bottom surface increases. For the linearly varying temperature on the left surface, the heat flux is lower near the bottom and higher near the mid portion of the surface from where hot fluids discharge into the core of the enclosure. However, the heat transfer is lowest near the top end where two surfaces meet with the same temperature. Heat transfer through the right surface shows that an increase in permeability accelerates heat transfer. However, the permeability has almost no effect on heat transfer through the portion where the temperature is linearly distributed. 

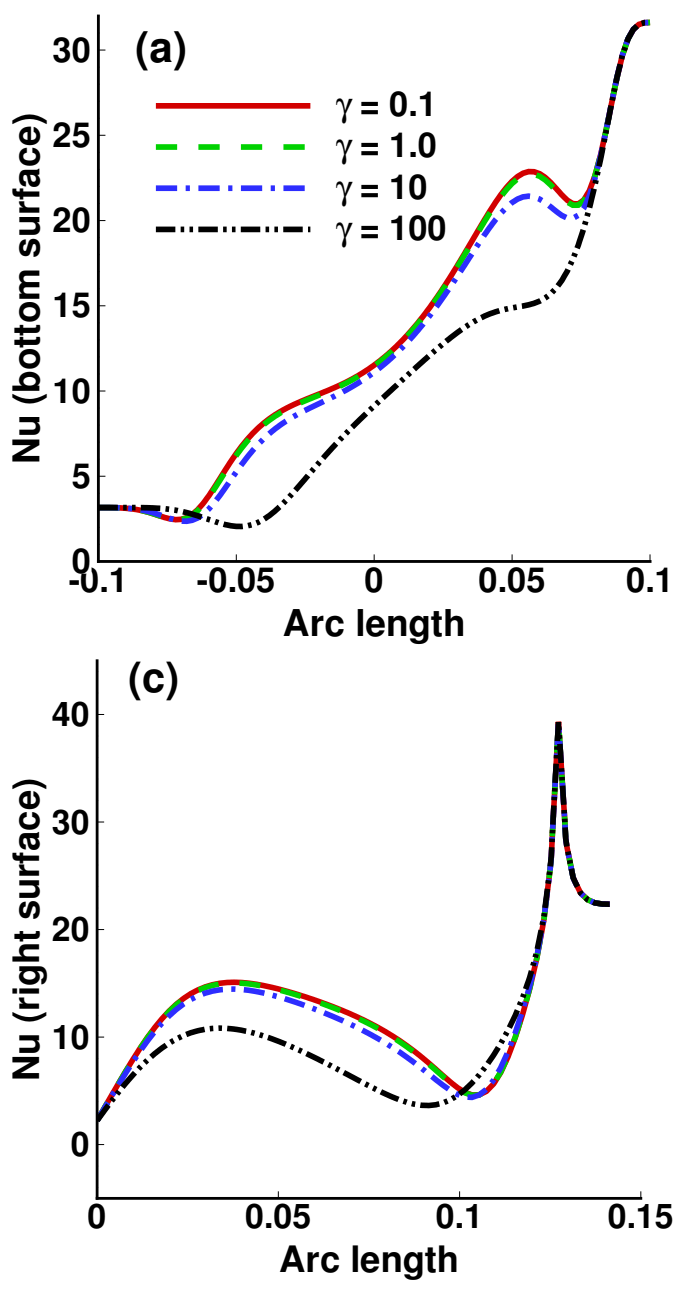

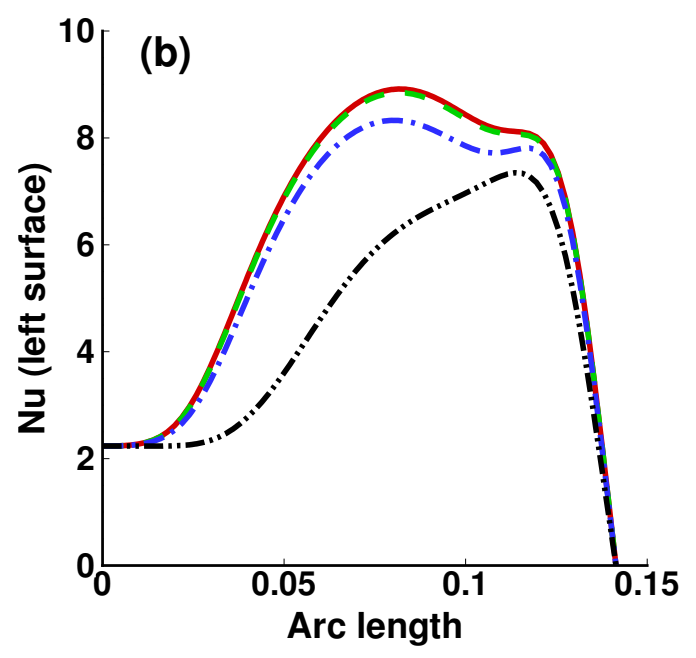

Figure 3: Rate of heat transfer from the surfaces for different values of $\gamma$ with $\mathrm{Ra}=10^{5}, \operatorname{Pr}=0.7, \lambda=0.0$ (a) bottom surface, (b) left surface and (c) right surface. 
We show the time series of average heat transfer through three surfaces of the enclosure for different values of heat generation parameter, $\lambda$, in Figure 4. Figure 4(a) shows the average heat transfer through the uniformly heated bottom surface. Initially, the heat transfer rate is very high for all values of $\lambda$ due to conduction. When the convection starts to dominate the heat transfer, we see undershoot followed by some oscillations. As the heat generation parameter increases, the heat transfer through this surface decreases which, as discussed before, is expected. The time evolution of average heat transfer on the left inclined surface, which is linearly heated, is shown in Figure 4(b). A large variation in heat transfer is seen for increasing values of $\lambda$ when the convection starts to dominant in the time period $0.02<\tau<0.04$. After that, the heat transfer reduces slightly and enters a steady state. Figure 4(c) shows the average heat transfer on the cold right inclined surface. There is a large variation in heat transfer during the steady state period for increasing values of the heat generation parameter.

\section{Conclusion}

We studied the effects of internal heat generation and porosity of the medium on the natural convection laminar flow and heat transfer in a triangular enclosure where one of the inclined surfaces is non-isothermal. We used a finite volume solution technique and applied the Boussinesq approximation to treat buoyancy effects. The studies were carried out for a fluid having a Prandtl number of 0.7 and a Rayleigh number of $10^{5}$. Increasing heat generation in the fluid reduces the thermal gradients near the heated bottom surface of the enclosure. The strength of the dominant vortex induced by buoyancy is reduced due to increasing internal heat generation, and a double vortex structure develops. Increasing the permeability of the medium for a fixed heat generation parameter value also increases heat transfer at the surfaces. 

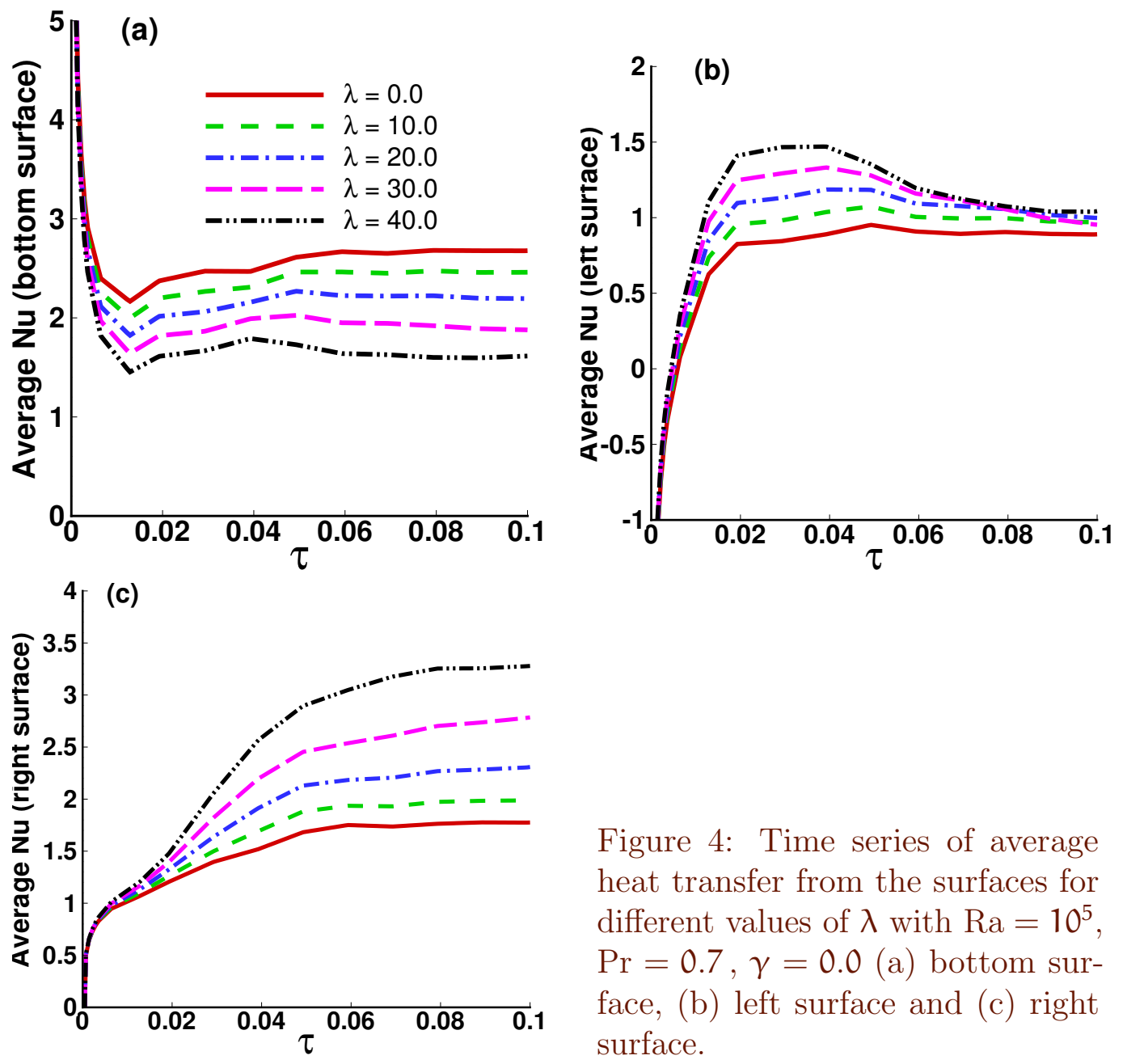

Figure 4: Time series of average heat transfer from the surfaces for different values of $\lambda$ with $\mathrm{Ra}=10^{5}$, $\operatorname{Pr}=0.7, \gamma=0.0$ (a) bottom surface, (b) left surface and (c) right surface. 


\section{References}

[1] Ostrach, S., Natural convection in enclosures, in: J.P. Hartnett, H. Irving (Eds.), Adv. Heat Transfer, 8 (1972) 161-227. doi:10.1016/S0065-2717(08)70039-X C128

[2] S. Ostrach, Natural convection in enclosures, ASME J. Heat Transfer, 110 (1988) 1175-1190. doi:10.1115/1.3250619 C128

[3] G. Shiralkar, C. Tien, A Numerical study of the effect of a vertical temperature difference imposed on a horizontal enclosure, Numer. Heat Transfer 5 (1982) 185-197. doi:10.1080/10407798208547013 C128

[4] M. November, M. W. Nansteel, Natural convection in a rectangular enclosure heated from below and cooled along one surface, Internat. J. Heat Mass Transfer 30 (1987) 2433-2440. doi:10.1016/0017-9310(87)90233-X C128

[5] J. D. Nicolas, M. W. Nansteel, Natural convection in a rectangular enclosure with partial heating on the lower surface; experimental results, Int. J. Heat Mass Transfer 36 (1993) 4067-4071. doi:10.1016/0017-9310(93)90156-Z C128

[6] M. M. Ganzarolli, L. F. Milanez, Natural convection in rectangular enclosures heated from below and symmetrically cooled from the sides, Int. J. Heat Mass Transfer 38 (1995) 1063-1073. doi:10.1016/0017-9310(94)00217-J C128

[7] K. Velusamy, T. Sundarajan, K. N. Seetharamn, Laminar natural convection in an enclosure formed by non-isothermal walls, in: Proc. 11th Internat. Conf. Heat Transfer, Korea, 3 (1998) 459-464. C128

[8] M. A. Hossain, M. Wilson, Natural convection flow in a fluid-saturated porous medium enclosed by non-isothermal walls with heat generation, Int. J. Therm. Sci., 41 (2002) 447-454. doi:10.1016/S1290-0729(02)01337-6 C128, C131 
[9] T. Basak, S. Roy, C. Thirumalesha, Finite element analysis of natural convection in a triangular enclosure: Effects of various thermal boundary conditions, Chemical Engineering Science, 62 (2007) 2623-2640. doi:10.1016/j.ces.2007.01.053 C129

[10] P. Cheng, Heat transfer in geothermal systems, Adv. Heat Transfer 14 (1979) 1-105. doi:10.1016/S0065-2717(08)70085-6 C129

[11] S. C. Saha, M. M. K. Khan, A review of natural convection and heat transfer in attic-shaped space, Energy and Buildings, 43 (2011) 2564-2571. doi:10.1016/j.enbuild.2011.06.020 C129

[12] A. Bejan, D. Poulikakos, Natural convection in an attic shaped space filled with porous material, J. Heat Transfer, 104 (1982) 241-247. doi:10.1115/1.3245079 C129

[13] Y. Varol, H. F. Oztop, A. Varol, Free convection in porous media filled right-angle triangular enclosures, Int. Comm. Heat Mass Trans., 33 (2006) 1190-1197. doi:10.1016/j.icheatmasstransfer.2006.08.008 C129

[14] Y. Varol, H. F. Oztop, A. Varol, Effects of thin fin on natural convection in porous triangular enclosures, Int. J. Therm. Sci., 46 (2007) 1033-1045. doi:10.1016/j.ijthermalsci.2006.11.001 C129

[15] Y. Varol, H. F. Oztop, A. Varol, Natural convection in porous triangular enclosures with a solid adiabatic fin attached to the horizontal wall, Int. Comm. Heat Mass Trans., 34 (2007) 19-27.

doi:10.1016/j.icheatmasstransfer.2006.09.004, C129

[16] S. C. Saha, Y. T. Gu, M. M. Molla, S. Siddiqa, M. A. Hossain, Natural convection from a vertical plate embedded in a stratified medium with uniform heat source, Desalination and Water Treatment, 44 (2012) 7-14. doi:10.1080/19443994.2012.691696 C130 


\section{Author addresses}

1. Suvash C. Saha, School of Chemistry, Physics and Mechanical Engineering, Queensland University of Technology, Brisbane, Queensland 4001, Australia. mailto:suvash.saha@qut.edu.au

2. Y. T. Gu, School of Chemistry, Physics and Mechanical Engineering, Queensland University of Technology, Brisbane, Queensland 4001, Australia.

mailto:yuantong.gu@qut.edu.au 